\title{
Untersuchungen zur Möglichkeit der Empfindlichkeitstestung von Mykobakterien mit dem MB/BacT-Kultursystem ${ }^{1}$
}

\author{
Investigations About the Possibility for Testing the Susceptibility of Mycobacteria With the \\ MB/BacT Culture System ${ }^{1}$
}

J. Beer ${ }^{2,3}$, R. Küchler ${ }^{4}$, A. C. Rodloff ${ }^{2}$

Zusammenfassung: Es wurde eine Methode zur Empfindlichkeitstestung von Mykobakterien in Flüssigkulturmedien ohne Verwendung radioaktiver Isotope entwickelt: Kommerziell erhältliche MB/BacT-Kulturflaschen wurden mit geeigneten Konzentrationen von Antituberkulotika versetzt, anschließend mit einer Mykobakterien-Testsuspension (MacFarland Nr. 1) beimpft und im MB/BacT-System bebrütet. Bei der Untersuchung von Pyrazinamid (PZA) wurde außerdem der $\mathrm{pH}$-Wert in den Kulturflaschen mit $\mathrm{KH}_{2} \mathrm{PO}_{4}$ auf 5,5 abgesenkt. Eine Antituberkulotika-freie Kulturflasche, die mit der 1:100 verdünnten Testsuspension beimpft wurde, diente als Kontrolle (1\%-Kontrolle). Wenn vom MB/BacT-System in dieser Kontrollflasche Wachstum erkannt wurde (nach ca. 7 Tagen), wurde die Untersuchung beendet und der bis zu diesem Zeitpunkt in den Antituberkulotika-haltigen Kulturflaschen festgestellte Wachstumsstatus bewertet. Für ausgewählte $M$. tuberculosis-Stämme wurden die MHKWerte folgender Antituberkulotika bestimmt: Ethambutol (EMB), Isoniazid (INH), Rifampicin (RMP), Streptomycin (SM), Dihydrostreptomycin, Protionamid und PZA. Die Testung von $16 \mathrm{M}$. tuberculosis-Stämmen und 3 Stämmen atypischer Mykobakterien gegenüber EMB (2,5 mg/l), INH (1,0 mg/l), RMP (2,0 $\mathrm{mg} / \mathrm{l}), \mathrm{SM}(2,0 \mathrm{mg} / \mathrm{l})$ und PZA $(50 \mathrm{mg} / \mathrm{l})$ ergab die gleichen Empfindlichkeitsbewertungen, wie sie mit Referenzmethoden (Verdünnungstest mit LöwensteinJensen-Medium, BACTEC 460TB) erhalten wurden.

Schlüsselwörter: Mikrobielle Empfindlichkeitstestung/Methodik; Mycobacterium tuberculosis/Medikamenteneffekte; Antituberkulotika.

\footnotetext{
${ }^{1}$ Teile dieser Arbeit wurden auf der 48. Jahrestagung der Deutschen Gesellschaft für Hygiene und Mikrobiologie, Bonn, 8.-11.10.1996, vorgestellt

2Institut für Medizinische Mikrobiologie und Infektionsepidemiologie, Universität Leipzig

3Korrespondenzadresse: Dr. Jörg Beer, Institut für Medizinische Mikrobiologie und Infektionsepidemiologie, Universität Leipzig, Liebigstraße 24, D-04103 Leipzig. Fax: +49-341-9715209

${ }^{4}$ Institut für Mikrobiologie und Serologie, Krankenhaus Neukölln, Berlin

Eingegangen: 27. Februar 1997 / Angenommen: 6. Juni 1997
}

Summary: A method for susceptibility testing of mycobacteria using isotope-free fluid culture media was developed. Commercially available $\mathrm{MB} / \mathrm{BacT}$ process bottles supplemented with antituberculotics in adequate concentrations were inoculated with a mycobacteria test suspension (MacFarland No.1) and loaded into the system. In case of testing pyrazinamide (PZA), the medium in the culture bottles was acidified to $\mathrm{pH} 5.5$ by $\mathrm{KH}_{2} \mathrm{PO}_{4}$. A bottle without antituberculotics inoculated with a 1:100 dilution of the test suspension served as a control ( $1 \%$ control). At the time the MB/BacT recognized growth in the control bottle, the test was terminated (after approximately 7 days) and the growth status of the bottles containing antituberculotics was determined. The minimum inhibitory concentrations of ethambutol (EMB), isoniazid (INH), rifampicin (RMP), streptomycin (SM), dihydrostreptomycin, prothionamid, and PZA were determined for selected strains of $M$. tuberculosis. The susceptibility testing of 16 strains of $M$. tuberculosis and 3 mycobacteria other than tubercle bacilli to EMB (2.5 $\mathrm{mg} / \mathrm{l}), \mathrm{INH}(1.0 \mathrm{mg} / \mathrm{l}), \mathrm{RMP}(2.0 \mathrm{mg} / \mathrm{l}), \mathrm{SM}(2.0 \mathrm{mg} / \mathrm{l})$, and PZA ( $50 \mathrm{mg} / \mathrm{l})$ exhibited the same susceptibility patterns as standard methods (dilution method with Löwenstein-Jensen medium, BACTEC 460 TB).

Keywords: Microbial Sensitivity Tests/methods; Mycobacterium tuberculosis/drug effects; Antitubercular Agents.

$\mathrm{D}_{\mathrm{s}}$ er Einsatz von Flüssigkulturen im MykobakterienLabor hat nicht nur das schnellere Erkennen positiver Proben ermöglicht [1-9], sondern auch zu einer Beschleunigung der Empfindlichkeitstestung von Mykobakterien gegenüber Antituberkulotika geführt [1014]. Das Auftreten von multiresistenten $M$. tuberculosis-Stämmen hat das Interesse an einer möglichst schnellen Empfindlichkeitstestung besonders gefördert. Neben der konventionellen, langwierigen, mit Oberflächenkulturen arbeitenden Proportionsmethode gilt inzwischen auch die Testung mit dem mit Flüssigkulturen arbeitenden System BACTEC 460TB (Becton Dickinson and Company) als Standardmethode bei 
der Empfindlichkeitstestung von Tuberkuloseerregern. Das System BACTEC 460TB nutzt allerdings ein radiometrisches Meßprinzip, was einer breiten Anwendung hinderlich ist. Es gibt deshalb Bemühungen, andere Nachweissysteme für die Empfindlichkeitstestung von Mykobakterien zu etablieren [15-25]. In der vorliegenden Arbeit haben wir die Möglichkeit geprüft, mit dem für die Mykobakteriendiagnostik entwickelten Kultursystem MB/BacT (Organon Teknika Corp.) die Empfindlichkeit von Mykobakterien gegenüber verschiedenen Antituberkulotika zu bestimmen.

\section{Material und Methoden}

\section{Mykobakterienstämme}

Die untersuchten Mykobakterienstämme entstammen den Stammsammlungen des Instituts für Mikrobiologie und Serologie am Krankenhaus Neukölln (Patientenstämme mit K-Nummern bezeichnet) und des Instituts für Medizinische Mikrobiologie und Infektionsepidemiologie der Universität Leipzig (bezeichnet mit Patienteninitialen). Außerdem wurden Stämme von Ringversuchen zur Empfindlichkeitsprüfung von Mykobakterien, die das Institut für Standardisierung und Dokumentation in Medizinischen Laboratorien e.V. (INSTAND e.V.) in Zusammenarbeit mit dem Arbeitskreis Mykobakterien organisiert, in die Untersuchungen einbezogen (bezeichnet mit RV96- und RV97-Nummern).

\section{Antituberkulotika}

\section{a) Untersuchungen mit dem $M B / B a c T$}

Wenn nicht anders angegeben, wurden für die Untersuchungen mit dem MB/BacT von Fatol Arzneimittel $\mathrm{GmbH}$ zur Verfügung gestelltes EMB, INH, PTH, PZA, RMP und SM (als Streptomycinsulfat) verwendet. DHS (als Sesquisulfat) wurde von Sigma-Aldrich Chemie $\mathrm{GmbH}$ bezogen. Zu Vergleichszwecken wurde außerdem der Arneimittelkit BACTEC S.I.R.E. von Becton Dickinson and Company genutzt. Von RMP (Fatol Arzneimittel GmbH) und PTH wurden 100fach konzentrierte Stammlösungen in Dimethylformamid hergestellt. Die weiteren-Verdünnungen erfolgten mit aqua dest., so daß die Endkonzentration von Dimethylformamid in den Versuchsansätzen maximal bei $0,04 \%$ lag. Alle anderen Antituberkulotika wurden in aqua dest. gelöst. Die Antituberkulotika-Lösungen wurden sterilfiltriert. Zur MHK-Bestimmung wurden geometrische Verdünnungsreihen der Antituberkulotika eingesetzt. . Folgende Konzentrationsbereiche wurden untersucht (Angaben in mg/l): EMB: 0,13-2,0; INH: 0,031-2,0; PTH: 0,25-4,0; RMP: 0,016-2,0; SM: 0,063-4; DHS: 0,063-1,0; PZA: 12,5-100. Für die Empfindlichkeitstestung bei einer einzigen Antituberkulotikum-Konzentration wurden folgende Konzentra-

Nicht standardisierte Abkürzungen: DHS, Dihydrostreptomycin; EMB, Ethambutol; INH, Isoniazid: LJ - Löwenstein-Jensen-Medium; MHK. Minimale Hemmkonzentration; PTH. Protionamid; PZA.Pyrazinamid; RMP, Rifampicin; SM, Streptomycin. tionen genutzt (mg/l): EMB: 2,5; INH: 1,0; RMP: 2,0; SM: 2,0; PZA: 50.

\section{b) Untersuchungen mit dem BACTEC 460TB}

Es wurde entsprechend den Vorschriften des Herstellers mit dem Arzneimittelkit BACTEC S.I.R.E. gearbeitet; die Testkonzentrationen waren (Angaben in mg/l): EMB: 2,5; INH: 0,1; RMP: 2,0; SM: 2,0; PZA: 100.

\section{c) LJ-Verdünnungstest}

Es wurden Antituberkulotika-haltige Löwenstein-Jensen-Kulturmedien von der Biologische Analysensystem $\mathrm{GmbH}$ genutzt; die kritischen Konzentrationen waren (mg/l): EMB: 1,0; INH: 0,25; RMP: 32; SM: 4,0; PZA: 256.

\section{Empfindlichkeitstestung mit dem MB/BacT}

a) Arbeitsprinzip des $M B / B a c T$

Das $\mathrm{MB} / \mathrm{BacT}$ ist ein automatisiertes MykobakterienKultursystem, das sowohl Flüssigkultur-Flaschen bebrütet als auch das Wachstum in den Flaschen mißt, analysiert und den aktuellen Wachstumsstatus jeder Flasche als positiv bzw. negativ ausweist. Wachstum in den Flaschen wird vornehmlich über das bei Stoffwechselprozessen freiwerdende $\mathrm{CO}_{2}$ erkannt. Das $\mathrm{CO}_{2}$ bewirkt bei dem am Boden der Kulturflaschen befindlichen $\mathrm{pH}$-Indikatoren-Gemisch Farbänderungen, die im 10-Minuten-Takt reflexionsphotometrisch gemessen und als Reflexionseinheiten angegeben werden.

\section{b) Kulturmedium und -bedingungen beim $M B / B a c T$}

Die MB/BacT-Kulturflaschen sind hermetisch verschlossene Flaschen mit einem Gesamtvolumen von $70 \mathrm{ml}$. Beimpfungen erfolgen durch ein Gummiseptum..Die Flaschen sind mit jeweils $10 \mathrm{ml}$ eines angereicherten Middlebrook 7H9-Mediums gefüllt. Die Atmosphäre in der Flasche ist eine spezielle Kohlendioxid-Sauerstoff-Mischung. Die Flaschen werden ohne zu schütteln bei $36^{\circ} \mathrm{C}$ bebrütet.

\section{c) Herstellung der Mykobakterien-Testsuspensionen}

Die Mykobakterien-Testsuspensionen wurden auf zweierlei Weise erhalten: Suspension einer frischen Oberflächenkultur in aqua dest. ster. und Einstellung auf eine Keimdichte von MacFarland 1,0 oder Einstellung einer frischen positiven MB/BacT-Kultur auf ein Wachstumsinkrement von 200 Reflexionseinheiten, was einer Keimdichte von MacFarland 1,0 entspricht. Bei frischen Kulturen zeigt sich eine gute Korrelation zwischen der Keimdichte und den vom MB/BacT gemessenen Reflexionseinheiten.

\section{d) Standard-Untersuchungsansätze}

Die mit jeweils $0,5 \mathrm{ml}$ Rekonstitutionsmedium (dient bei diagnostischem Einsatz der Kulturflaschen zum Auflösen des Antibiotika-Supplements) versetzten Kulturflaschen wurden wie folgt beimpft. Wachstumskontrolle: $0,5 \mathrm{ml}$ Mykobakterien-Testsuspension + 0,5 $\mathrm{ml}$ aqua dest. ster. 1\%-Kontrolle: $0,5 \mathrm{ml}$ 1:100 
vcrdiunntc Mykobakterien-Testsuspension (Verdünnung mit aqua dest. ster.) $+0,5 \mathrm{ml}$ aqua dest. ster. Antituberkulotika-Ansïitze: $0,5 \mathrm{ml}$ MykobakterienTestsuspension $+0,5 \mathrm{ml}$ Antituberkulotika-Lösung (mit ciner Konzentration, die z.u der gewünschten Endkonzentration in cler Kulturflasche führt).

\section{c) Untersuchungsantätze zur Testung von PZA}

Im Unterschied $\% u$ den Standard-Untersuchungsansätzen wurden alle zur Testung benötigten Kulturflaschen (einschließlich Kontrollen) zwecks Absenkung des $\mathrm{pH}$-Wertes auf $5,5 \mathrm{mit} 2,0 \mathrm{ml}$ steriler, wäßriger 1 molarer $\mathrm{KH}_{2} \mathrm{PO}_{4}$-Lösung versetzl.

\section{f) Ausw'ertung der Untersuchungsansätze}

Dic Untersuchungsansäitze wurden im MB/BacT-System so lange untersucht, bis in der $1 \%$-Kontrolle vom $\mathrm{MB} / \mathrm{BacT}$ Wachstum erkannt wurde. Der zu diesem Zeitpunkt vom MB/BacT festgestellte Wachstumsstatus für die Antituberkulotika-haltigen Ansätze wurde als Endergebnis bezüglich des Wachstums in diesen Ansätzen betrachtet.

\section{Empfindlichkeitstestung mit dem BACTEC 460 TB} Die Untersuchungen wurden entsprechend den Vorschriften des Herstellers $[26,27]$ durchgeführt.

Empfindlichkeitstestung mit dem Verdünnungstest auf Löwenstein-Jensen-Medium (LJ-Verdünnungstest)

Die Untersuchungen der Empfindlichkeiten gegenüber EMB, INH, RMP und SM wurden entsprechend DIN 58 943, Teil 8 durchgeführt [28]. Die Empfindlichkeitstestung von PZA erfolgte entsprechend den Empfehlungen des Deutschen Zentralkomitees zur Bekämpfung der Tuberkulose [29].

\section{Ergebnisse}

Zunächst wurden für den Stamm $M$. tuberculosis H37Rv, der sich durch eine hohe AntituberkulotikaEmpfindlichkeit auszeichnet, die MHK-Werte von EMB, INH, RMP, SM, DHS, PTH und PZA ermittelt.

In Abbildung 1 sind die Kurvenverläufe eines für MHK-Bestimmungen repräsentativen Versuchs dargestellt, bei dem die MHK von SM bestimmt wurde. Es zeigte sich, daß bis zum Zeitpunkt der Positivmeldung der 1\%-Kontrolle die Kulturflaschen mit SM-Konzentrationen bis $0,13 \mathrm{mg} / \mathrm{l}$ als positiv erkannt wurden, $a b$ einer Konzentration von $0,25 \mu \mathrm{g} \mathrm{SM} / \mathrm{ml}$ vom $\mathrm{MB} / \mathrm{BacT}$ aber kein Wachstum festgestellt wurde. Die MHK von SM wurde folglich mit $0,25 \mathrm{mg} / \mathrm{l}$ bestimmt. Erwartungsgemäß zeigte sich auch, daß subinhibitorische SM-Konzentrationen eine Verlangsamung des Mykobakterien-Wachstums bewirken. In allen untersuchten Fällen konnten - wie am Beispiel demonstriert - die MHK-Werte durch die vom MB/BacT ermittelte Wachstumsanalyse unproblematisch festgelegt werden. Nur in seltenen Fällen wurde beobachtet, daß An- tituberkulotika-haltige Ansätze kurz nach dem Positivwerden der $1 \%$-Kontrolle als positiv erkannt wurden. Wenn die Positivmeldungen für die Antituberkulotikahaltigen Ansätze in zeitlicher Nähe zum Versuchsende liegen, kann es zufallsbedingt zu Abweichungen des MHK-Wertes um eine geometrische Stufe kommen.

In Tabelle 1 sind die auf der dargestellten Weise ermittelten MHK-Werte ausgewählter Antituberkulotika für den Stamm $M$. tuberculosis H37Rv ausgewiesen. Folgende MHK-Werte $(\mathrm{mg} / \mathrm{l})$ sind für diesen Stamm typisch: EMB - 1,0; INH - 0,25; PTH - 1,0; RMP 0,031 ; SM - 0,25; DHS - 0,50; PZA - 25. Für den PZA-resistenten Stamm $M$. bovis BCG wurde für PZA ein MHK-Wert von $\geq 100 \mathrm{mg} / \mathrm{l}$ bestimmt. Wie in Tabelle 2 dargestellt, wurden für 6 weitere $M$. tuberculosis-Stämme die MHK von INH bestimmt. INH-sensible Stämme (L.W., K304, K424, K1151) wiesen Werte zwischen 0,13 und $0,50 \mathrm{mg} / \mathrm{l}$ auf, die MHK-Werte INH-resistenter Stämme lag bei $\geq 2,0 \mathrm{mg} / 1$.

Die Testung von PZA erfordert eine Absenkung des $\mathrm{pH}$-Wertes des Kulturmediums auf $\leq 6,0$. Durch Zugabe von 2,0 ml 1 molarer $\mathrm{KH}_{2} \mathrm{PO}_{4}$-Lösung konnte der $\mathrm{pH}$-Wert auf 5,5 abgesenkt werden (Abbildung 2). Die Zugabe von $\mathrm{KH}_{2} \mathrm{PO}_{4}$-Lösung kann in Abhängigkeit vom untersuchten Stamm zu einer Verzögerung des Wachstums in den Kulturflaschen führen, die Untersu-

Tabelle 1 Mit dem MB/BacT bestimmte MHK-Werte von verschiedenen Antituberkulotika für $M$. tuberculosis H37Rv. Bei Angabe mehrerer MHK-Werte handelt es sich um Ergebnisse unabhängiger Versuche. Mit * markierte Werte wurden bei Verwendung von Wirkstoffen des S.I.R.E.-Arzneimittelkits erhalten.

\begin{tabular}{ll} 
Antituberkulotikum & $\begin{array}{l}\text { MHK } \\
(\mathrm{mg} / \mathrm{l})\end{array}$ \\
\hline EMB & $1,0 / 1,0 / 1,0$ \\
INH & $0,13 / 0,25^{\star} / 0,25$ \\
PTH & $>0,50 / 1,0$ \\
RMP & $\leq 0,031 / 0,016 * / 0,031$ \\
SM & $\leq 0,50 / 0,25 * / 0,25 / 0,5$ \\
DHS & 0,50 \\
PZA & 25
\end{tabular}

Tabelle 2 Mit dem MB/BacT bestimmte MHK-Werte von INH für verschiedene M.tuberculosis-Stämme. Bei Angabe mehrerer MHK-Werte handelt es sich um Ergebnisse unabhängiger Versuche. Mit * markierte Werte wurden bei Verwendung von Wirkstoffen des S.I.R.E.-Arzneimittelkits erhalten.

\begin{tabular}{ll}
\hline M. tuberculosis-Stamm & MHK von INH (mg/l) \\
\hline L.W. & 0,25 \\
K304 & $0,50^{\star}$ \\
K424 & $0,25 / 0,25^{*}$ \\
K917 & $\geq 2,0$ \\
K1151 & $\leq 0,13 / 0,25$ \\
K1316 & $\geq 2,0$ \\
\hline
\end{tabular}



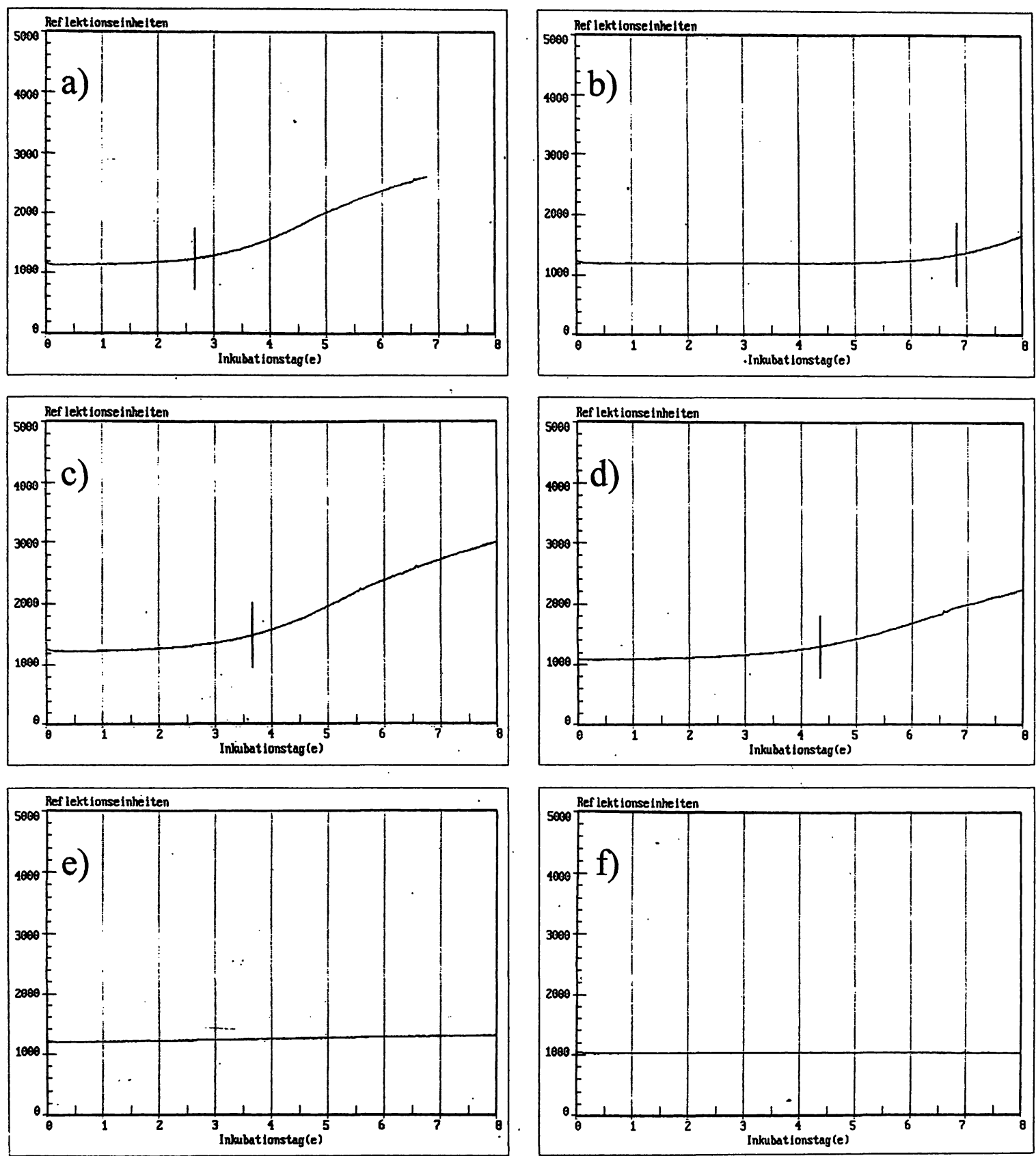

Abbildung 1 Wachstumskurven bei einem Versuch zur Bestimmung der MHK von SM für den Stamm M: tuberculosis H37Rv mit dem MB/BacT. Gezeigt sind Originalausdrucke des Gerätesystems, die den zeitlichen Verlauf der Entwicklung der Reflexionseinheiten für die ausgewählten Kulturflaschen zeigen. Der senkrechte Balken im Kurvenverlauf markiert den Zeitpunkt, an dem vom MB/BacT Wachstum

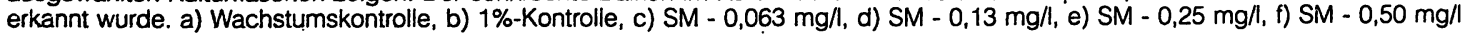

chung kann folglich etwas (1-2 Tage) länger dauern. Entsprechend den allgemeinen Erfahrungen wachsen einige Stämme bei diesem pH-Wert nicht mehr.

Die in den Tabellen 1 und 2 ausgewiesenen Ergebnisse von Mehrfachbestimmungen yermitteln einen Eindruck von der guten Reproduzierbarkeit der MHK-
Bestimmungen mit dem MB/BaciT. Außerdem wurde beim INH, RMP und SM gezeigt, daß die Wirkstoffe verschiedener Anbieter gleiche MHK-Werte aufweisen.

Für vergleichende Untersuchungen der Empfindlichkeit von Mykobakterien mit dem MB/BacT einer- 
Abbildung 2 Verănderung des $\mathrm{pH}$ Wertes des Kulturmediums in den $\mathrm{MB} / \mathrm{BacT}$-Flaschen durch Zugabe von $1 \mathrm{~mol} / \mathrm{KH}_{2} \mathrm{PO}_{4}$-Lösung (Dreifachbestimmung)

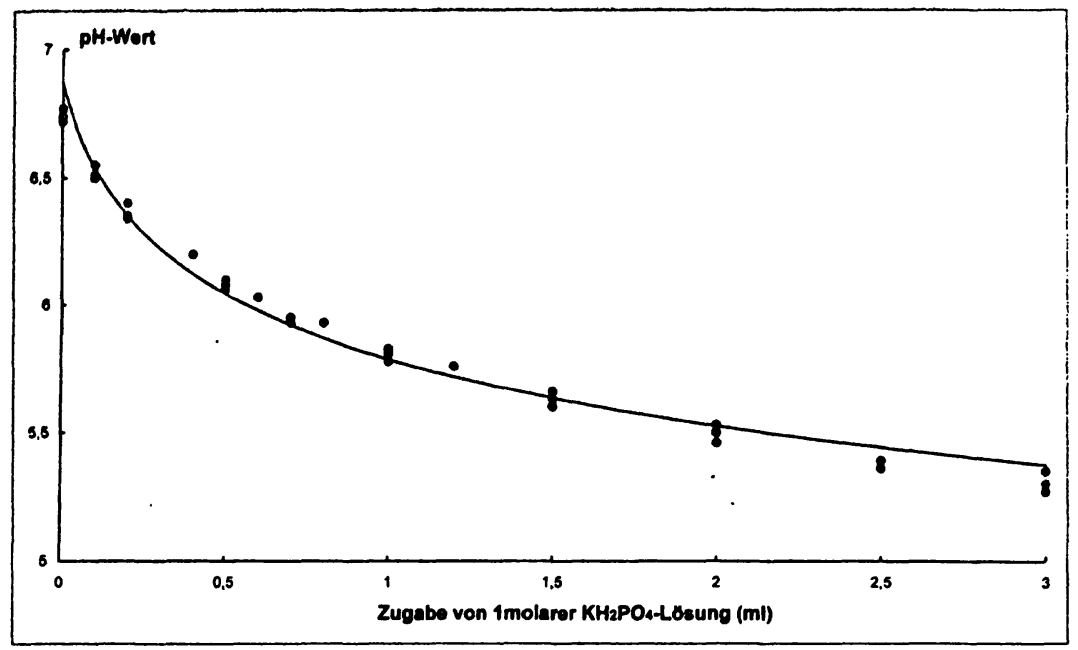

seits und dem LJ-Verdünnungstest bzw. dem BACTEC 460TB andererseits wurden für das MB/BacT folgende Testkonzentrationen festgelegt (Angaben in $\mathrm{mg} / \mathrm{l}$ ): EMB - 2,5; INH - 1,0; RMP - 2,0; SM - 2,0; PZA - 50. Bei der Auswahl dieser Konzentrationen wurden die für den Stamm M. tuberculosis H37RV bestimmten MHK-Werte sowie die beim BACTEC 460 benutzten Testkonzentrationen berücksichtigt. Wurde bei der genannten Konzentration des jeweiligen Antituberkulotikums im MB/BacT Wachstum festgestellt, so wurde der Stamm als resistent, bei fehlendem Wachstum als sensibel eingeschätzt.

Abbildung 3 zeigt am Beispiel des Stammes $M$. tuberculosis K852 die Kurvenverläufe für die Empfindlichkeitstestung gegenüber EMB, INH, RMP und SM.

In Tabelle 3 sind die Ergebnisse der vergleichenden Testung von $16 M$. tuberculosis-Stämmen und 3 Stämmen atypischer Mykobakterien zusammengefaßt. Es zeigt sich in allen Fällen eine Übereinstimmung der MB/BacT-Ergebnisse mit denen der Referenzmethoden. Von drei weiteren Stämmen liegen Einzelergebnisse vor, die nicht in Tabelle 3 erfaßt sind: die $M$. $t u$ berculosis-Stämme $\mathrm{K} 304$ und $\mathrm{K} 424$, die beim $\mathrm{MB} / \mathrm{BacT}$ als INH-sensibel einzuschätzen sind (siehe Tabelle 2), werden mit dem BACTEC 460 ebenfalls als INH-sensibel bewertet; der Stamm $M$. bovis BCG wurde mit dem MB/BacT zutreffend als PZA-resistent charakterisiert (MHK-Wert s.o.). Mit mehreren Stämmen wurden die Untersuchungen mit dem MB/BacT wiederholt, wobei identische Ergebnisse erzielt wurden.

\section{Diskussion}

Die vorgestellten Ergebnisse zeigen, daß es bei Nutzung der vorgestellten Methode mit dem MB/BacT möglich ist, die Antituberkulotika-Empfindlichkeit von Mykobakterien reproduzierbar zu charakterisie- ren. Bei der Ausarbeitung der Methode orientierten wir uns an den Arbeitsvorschriften für das BACTEC 460TB, die ihrerseits die Grundlagen der Proportionsmethode berücksichtigen. Dementsprechend nutzten wir als Bezugswert zur Beurteilung des Wachstums von Mykobakterien in den Antituberkulotika-haltigen Untersuchungsansätzen einen Kontrollansatz (1\%Kontrolle), dessen Mykobakterien-Inokulum nur ein hundertstel des Inokulums in den Test-Ansätzen betrug. Hat ein Antituberkulotika-haltiger Ansatz zum Zeitpunkt, an dem das MB/BacT bei der 1\%-Kontrolle Wachstum erkennt, noch einen negativen Wachstumsstatus, so kann davon ausgegangen werden, daß in diesem Ansatz weniger als $1 \%$ des MykobakterienInokulums vermehrungsfähig ist.

Das Inokulum der Mykobakterien-Testsuspension wurde so bemessen, daß die $1 \%$-Kontrolle bei $M$. $t u$ berculosis-Stämmen nach ca. 7 Tagen vom MB/BacT als positiv erkannt wurde. Ein derartiges Inokulum liegt i.d.R. vor, wenn eine Mykobakterienkultur vom MB/BacT positiv gemeldet wird. Die MykobakterienTestsuspension kann aber auch nach entprechender Verdünnung aus einer $\mathrm{MB} / \mathrm{BacT}$-Kulturflasche gewonnen werden, die noch mehrere Tage nach dem Positivwerden im MB/BacT-System bebrütet wurde. Es ist also möglich, sofort nach dem Positivwerden einer MB/BacT-Kultur die Empfindlichkeitsprüfung zu starten, andererseits ist auch die Bearbeitung einer Kultur möglich, die beispielsweise an arbeitsfreien Tagen positiv gewordèn ist. Schließlich kann die Empfindlichkeitstestung auch mit einem auf festen Nährböden gewachsenen Stamm durchgeführt werden. Eine zu geringe Keimzahl des Inokulums scheint nach bisheriger, begrenzter Erfahrung - abgesehen von der längeren Untersuchungszeit - nicht das Ergebnis der Empfindlichkeitstestung zu beeinflussen.

Um methodisch bedingte Abweichungen der Inokulumgröße zu berücksichtigen, schien es zweckmäßig. die Antituberkulotika-haltigen Testansätze noch weitere 24 Stunden nach dem Positivwerden der 1\%-Kon- 
trolle zu bebrüten und dann erneut zu bewerten. Unsere bisherigen Erfahrungen zeigten jedoch, daß sich dadurch bei den zur Routinetestung ausgewählten Antituberkulotika-Konzentrationen keine Veränderungen in der Beurteilung ergaben.

Die Wachstumskontrollen mit dem unverdünnten Test-Inokulum wurden bei den gewählten Testbedingungen nach ca. 3 Tagen positiv gemeldet. Nach dem Vorliegen ausreichender Erfahrungen mit der vorgestellten Methode kann möglicherweise auf diese Kontrolle verzichtet werden.

Für die Mykobakterien-Testsuspension und die zuzugebenden Antibiotikalösungen wurden mit $0,5 \mathrm{ml}$ bewußt relativ große Volumina ausgewählt, damit die bei der Zugabe mittels Spritzen nicht vermeidbaren Toleranzen relativ gering blieben. Die für die PZA-Testung erforderliche $\mathrm{pH}$-Absenkung im Kulturmedium ist durch das relativ große Volumen der zuzugebenden $\mathrm{KH}_{2} \mathrm{PO}_{4}$-Lösung sehr gut reproduzierbar.

Die ermittelten MHK-Werte für den Stamm M. tuberculosis H37Rv liegen in dem erwarteten Bereich [28, 30-33]; allerdings findet man in der Literatur unterschiedliche Angaben und wegen methodischer Unterschiede sind Vergleiche nur bedingt möglich. Besonders anzumerken ist die Anwesenheit von Tween 80 (Endkonzentration 0,02\%) in den $\mathrm{MB} /$ BacT-Test- flaschen, von dem bekannt ist, daß es in Abhängigkeit vom antimykobakteriellen Wirkstoff $\mathrm{zu}$ einer $\mathrm{Er}$ höhung oder Erniedrigung der MHK-Werte führen kann $[34,35]$. Die MHK-Werte von INH für die in den Tabellen 1 und 2 aufgeführten INH-sensiblen $M$. tuberculosis-Stämme H37Rv, K304, K424, K1151 und L.W. liegen zwischen 0,13 und $0,50 \mathrm{mg} / \mathrm{l}$, so da $\beta$ die beim BACTEC 460TB verwendeten INH-Konzentrationen von 0,1 oder $0,2 \mathrm{mg} / \mathrm{l}$ für das $\mathrm{MB} / \mathrm{BacT}$ nicht geeignet sind.

Das in der DIN-Empfehlung [28] empfohlene DHS zeigt im Rahmen der Fehlerbreite den gleichen MHKWert, wie das sonst bei den MB/BacT-Untersuchungen benutzte SM.

Um bei den Untersuchungen mit dem MB/BacT Ergebnisse zu erhalten, die den Resultaten der Referenzmethoden (LJ-Verdünnungstest bzw. BACTEC 460TB) entsprechen, können die beim BACTEC 460TB benutzten Antituberkulotika-Konzentrationen nicht einfach übernommen werden. Die zwischen den beiden Systemen bestehenden geringfügigen Unterschiede beim Kulturmedium, die unterschiedlichen atmosphärischen Bedingungen in den Kulturflaschen und die unterschiedlichen Methoden zur Messung des Einflusses von Antituberkulotika auf das Mykobakterienwachstum können zu differenten MHK-Werten beim

Tabelle 3 Vergleich der Ergebnisse von Empfindlichkeitstestungen von Mykobakterien mittels MB/BacT einerseits und BACTEC 460TB bzw. LJ-Verdünnungstest andererseits.

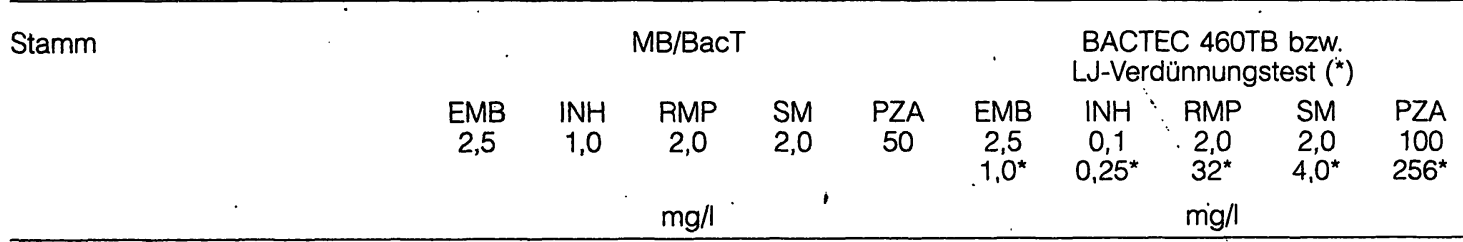

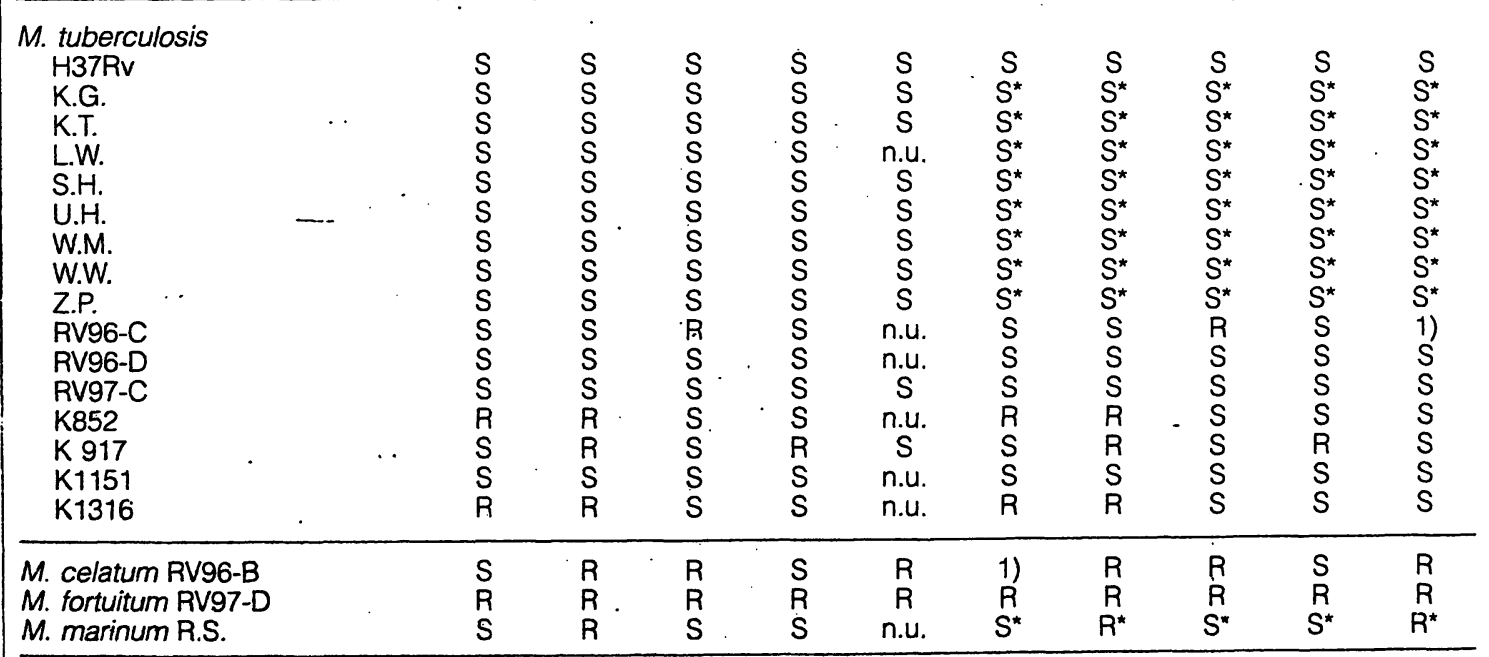

1) Bei der Charakterisierung des Stammes im Ringversuch (siehe Abschnitt "Material und Methoden") wurden unterschiedliche Ergebnisse erhalten. 

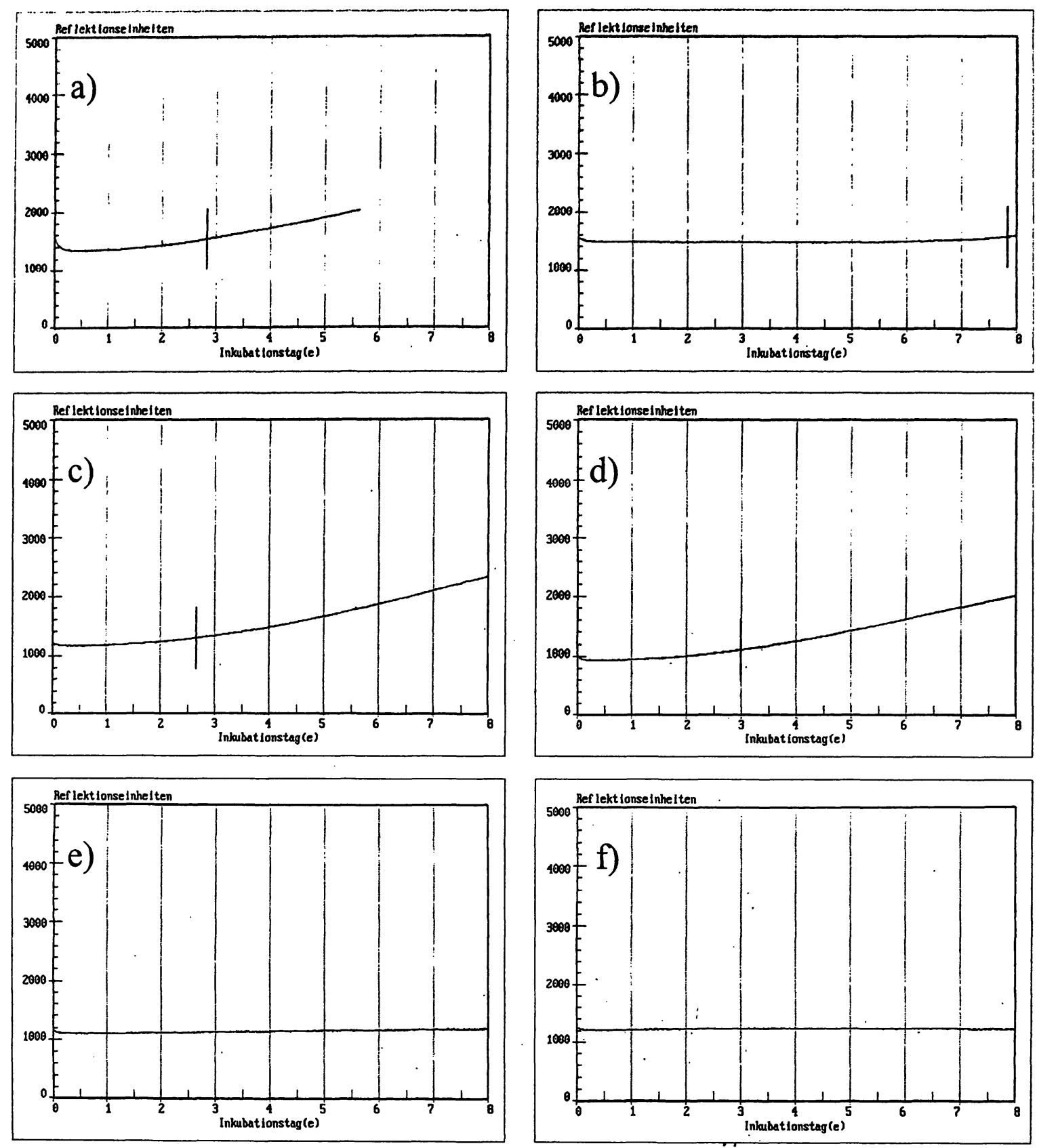

Abbildung 3 Wachtumskurven bei einem Versuch zur Empfindlichkeitstestung dés Stámmes $M$. tuberculosis K852 (EMB = resistent, $\mathrm{INH}=$ resistent, $\mathrm{RMP}=$ sensibel, $\mathrm{SM}=$ sensibel). a) Wachstumskontrolle, b) $1 \%$-Kontrolle, c) EMB - 2,5 mg/l, d) INH - 1,0 mg/l, e) RMP $-2,0 \mathrm{mg} / \mathrm{l}, \mathrm{f}) \mathrm{SM}-2,0 \mathrm{mg} / \mathrm{l}$

gleichen Stamm führen. Unter Berücksichtigung der ermittelten MHK-Werte und Beachtung der beim BACTEC 460TB gewählten Konzentrationen konnten Antituberkulotika-Testkonzentrationen gefunden werden, mit denen bei der Arbeit mit dem MB/BacT Ergebnissen erhalten wurden, die mit mit denen allgemein anerkannter Methoden (LJ-Verdünnungstest,
BACTEC 460TB) übereinstimmen. Ebenso wie bei der Testung mit dem BACTEC 460TB können innerhalb von ca. 7 Tagen verläßliche Aussagen über die Empfindlichkeit von Mykobakterienstämmen gemacht werden; in der Regel verlangt die Feststellung einer Resistenz nur einen Untersuchungszeitraum von 3-5 Tagen. 
Der bisherige Umfang der Untersuchungen erlaubt es noch nicht, die beim Methodenvergleich für das $\mathrm{MB} / \mathrm{BacT}$ ausgewählten Antituberkulotika-Konzentrationen verbindlich festzulegen. Das ist erst im Ergebnis einer umfangreicheren Methodenvalidierung möglich. Wenn sich resistente Stämme generell durch eine hohes MHK-Niveau auszeichnen - z.B. RMP-resistente Stämme [36], so ist die Festlegung der Testkonzentration wenig kritisch. Andernfalls hat die Wahl der Testkonzentration große Auswirkung auf die qualitative Bewertung der Empfindlichkeit. Bei den künftigen Untersuchungen zur Festlegung der Testkonzentrationen könnten darüber hinaus auch Grenzwerte für einen Intermediärbereich erarbeitet werden. Bei der vorliegenden Methode zur Empfindlichkeitstestung mit dem $\mathrm{MB} / \mathrm{BacT}$ kann die beim LJ-Verdünnungstest und BACTEC 460TB mögliche Einschätzung „Grenzwertig" nicht gegeben werden. Ensprechend dem Vorgehen bei vielen anderen Bakteriengruppen und der für Mykobakterien entwickelten resistance-ratio-Methode $[15,31,37]$ sollte eine breakpoint-Methode bei der Empfindlichkeitstestung von Mykobakterien möglich sein.

Ein Vorteil der Empfindlichkeitstestung mit dem MB/BacT besteht neben dem nichtradiometrischen Meßprinzip darin, daß nach dem Start der Untersuchung seitens des Untersuchers keine Aktivitäten erforderlich sind. Nach Meldung der Positivität der 1\%-Kontrolle kann der Test direkt abgelesen werden; die Untersuchungsdaten werden vom Gerät automatisch gespeichert. Diese außerordentlich effektive Testdurchführung und Auswertung - nach Bereitstellung entsprechender Software könnten die Testergebnisse automatisch vom $\mathrm{MB} / \mathrm{BacT}$ ermittelt und ausgedruckt werden - sollte die Einführung einer ausführlicheren Testung mit mehreren Konzentrationen pro Wirkstoff (s.o.) begünstigen.

Die bisherigen Untersuchungen wurden zum größten Teil mit $M$. tuberculosis-Stämmen durchgeführt. Die prinzipielle Möglichkeit, auch atypische Mykobakterien zu charakterisieren, wurde ebenfalls gezeigt.

\section{Danksagung}

Für die technische Unterstützung bei der Durchführung der Untersuchungen danken wir Frau E. Krawczyk und Frau B. Sakallah. Den Firmen Fatol Arzneimittel GmbH und Organon Teknika Corp. danken wir für die Bereitstellung von Testsubstanzen und Verbrauchsmaterial.

\section{Literatur}

1. Middlebrook G, Reggiardo Z, Tigertt, WD. Automatable radiometric detection of growth of Mycobacterium tuberculosis in selective media. Am Rev Respir Dis 1977;115:1067-9.

2. Fadda G, Roe SL. Recovery and susceptibility resting of $M$ )cobacterium tuberculosis from extrapulmonary specimens by the Bactec radiometric method. J Clin Microbiol 1984; 19:720-1.

3. Kirihara JM, Hillier SL, Coyle MB. Improved detection times for Mycobacterium avium complex and Mycobacterium tuberculosis with the BACTEC radiometric system. J Clin Mirobiol 1985;22:841-5.
4. Rüsch-Gerdes S, Schröder K-H, Finnern J. Untersuchungen mit dem system Bactec 460: 2. Isolierung von Mycobacterium tuberculosis aus Sputum. Vergleich der radiometrischen mit der konventionellen Methode. Prax Klin Pneumol 1987;41:219-22.

5. Anargyros P, Astill DSJ, LIM ISL. Comparison of improved BACTEC and Löwenstein-Jensen media for culture of mycobacteria from clinical specimens. J Clin Microbiol 1990;28:1288-91.

6. Naumann L. Die Leistungsfähigkeit von Flüssigkulturen bei der Untersuchung auf Mykobakterien. Pneumologie 1990:44:731-4.

7. D'Amato RF. Isenberg HD, Hochstein L, Mastellone AJ, Alperstein P. Evaluation of the Roche Septi-Chek AFB system for recovery of mycobacteria. J Clin Microbiol 1991:29:2906-8.

8. Stager CE, Libonati JP, Siddici SH, Davis JR, Hooper NM, Baker JF, Carter ME. Role of solid media when used in conjunction with the BACTEC system for mycobacterial isolation and identification. J Clin Microbiol 1991:29:154-7.

9. Luquin M. Gamboa F. Garcia Barcelo M, Manterola JM, Matas L. Gimenez M, Ausina V. Comparison of a biphasic non-radiometric system with Löwenstein-Jensen and Bactec-460 system for recovery of mycobacteria from clinical specimens. Tubercle Lung Dis 1996;77:449-53.

10. Snider Jr DE, Good RC, Kilburn JO, Laskowski Jr LF, Lusk RH, Marr JJ, Reggiardo Z. Middlebrook G. Rapid drug-susceptibility testing of Mycobacteria tuberculosis. Am Rev Respir Dis 1981;123:402-6.

11. Siddiqi SH, Libonati JP, Middlebrook G. Evaluation of a rapid radiometric method for drug susceptibility testing of Mycobacterium tuberculosis. J Clin Microbiol 1981;13:908-12.

12. Roberts GD, Goodman NL, Heifets L. Larsh HW, Lindner TH, McClatchy JK, McGinnis MR, Siddiqi SH, Wright P. Evaluation of the BACTEC radiometric method for recovery of mycobacteria and drug susceptibility testing of Mycobacterium tuberculosis from acid-fast smear-positive specimens. J Clin Microbiol 1983;18:68996.

13. Rüsch-Gerdes S, Schröder K-H, Fetting C. Untersuchungen mit dem System Bactec 460. Empfindlichkeitsprüfung bei Mycobacterium tuberculosis. Vergleich der radiometrischen mit der konventionellen Methode. Prax Klin Pneumol 1985;39:967-9.

14. Steadham JE, Stall SK, Simmank JL. Use of the Bactec system for the drug susceptibility testing of Mycobacterium tuberculosis, $M$. kansasii, and $M$. avium complex. Diagn Microbiol Infect Dis 1985;3:33-40.

15. Nilsson LE, Hoffner SE, Ansehǹ S. Rapid susceptibility testing of Mycobacterium tuberculosis by bioluminiscence assay of mycobacterial ATP. Antimicrob Agents Chemother 1988;32:1208-12.

16. Kawa DE. Pennell DR, Kubista LN. Schell RF. Development of a rapid method for determining the susceptibility of Mycobacterium tuberculosis to ioniazid using the gen-probe DNA hybridization system. Antimicrob Agents Chemother 1989;33:1000-5.

17. Zhan Y, Heym B, Alen B, Youg D, Cole S. The catalase-peroxidase gene and isoniazid resistance of Mycobacterium tuberculosis. Nature 1992;358:591-3.

18. Jacobs Jr WR, Barletta RG, Udani R, Chan J, Kalkut G, Sosne G, Kieser T, Sarkis GJ, Hatfull GF, Bloom BR. Rapid assessment of drug suscseptibilities of Mycobacterium tuberculosis by means of luciferase reporter phages.Science 1993;260:819-22.

19. Telenti A, Imboden $P$, Marchesi $F$, Schmidheini $T$, Bodmer $T$. Direct, automated detection of rifampin-resistant Mycobacteria tuberculosis by polymerase chain reaction and single-strand conformation polymorphism analysis. Antimicrob Agents Chemother 1993;37:2054-8.

20. Banerjee A, Dubnau E, Quemard A, Balasubramanian V, Um KS, Wilson T, Collins D, de Lisle G, Jacobs Jr WR. inhA, a gene encoding a target for isoniazid and ethionamide in Mycobacteritum tuberculosis. Science 1994;263:227-30.

21. Conville PS, Witebsky FG, MacLowry J. Antimicrobial susceptibilities of mycobacteria as determined by differential light scattering and correlation with results from multiple reference laboratories. J Clin Microbiol 1994;32:1554-9.

22. Norden MA, Kurzynski TA. Bownds SE, Callister SM, Schell RF. Rapid susceptibility testing of Mycobacterium tuberculosis (H37Ra) by flow cytometry. J Clin Microbiol 1995;33:1231-7.

23. Miyamoto J, Koga H, Kohno S, Tashiro T, Hara K. New drug susceptibility test for Mycobacterium tuberculosis using the hybridization protection assay. J Clin Microbiol 1996:34:1323-6.

24. Palaci M, Mizuka Ueki SY, Nakamura Sato D, Da Silva Telles MA, Curcio M, Matheus Silva EA. Evaluation of mycobacteria 
grow'th indicator tube for recovery and drug susceptibility testing of Micobacterium tuberculosis isolates from respiratory specimens. J Clin Microbiol 1996:34:762-4.

25. Walters SB, Hanna BA. Testing of susceptibility of Mycobucterium nuberculosis to isoniacid and rifampin by mycobacterium growilh indicator lube method. J Clin Microbiol 1996:34:1565-7.

26. Siddiqi SH. (Hrsg.) Bactec 460 TB-System. Handbuch und Arbcitsanleitung für Gerät und Produktc. Towson (Maryland): Johnston Laboratorics, 1987.

27. Arbeitsanleitung BACTEC S.I.R.E. Arzncimittelkit. Sparks (Maryland): Becton Dickinson and Company, 1995.

28. Empfindlichkcitsprüfungen von Tuberkulosebakterien gegen Chemotherapeutika. DIN 58943, Tcil 8. In: DIN Deutsches.Institut für Normung e.V., Hrsg. Medizinische Mikrobiologie und Immunologie. 2. Auflage. Berlin (Deutschland): Beuth Verlag, 1992:258-65. 29. Empfehlungen zur Methodik und Bewertung von Empfindlichkeitsprüfungen von Mykobakterien. Ärztl Lab 1973;19:227-84.

30. Wallace Jr RJ, Nash DR, Steele LC, Steingrube V. Susceptibility testing of slowly growing mycobacteria by a microdilution MIC method with 7H9 broth. J Clin Microbiol 1986;24:976-81. 31. Mor N, Simon B, Mezo N, Heifets L. Comparison of activities of rifapentine and rifampin against Mycobacterium tuberculosis residing in human macrophages. Antimicrob Agents Chemother 1995;39:2073-7.
32. Inderlied CB. Antimycobacterial agents: In vitro susceptibility testing, spectrum of activity, mechanisms of action and resistance. and assays for activity in biological fluids. In: Lorian V, editor. Antibiotics in laboratory medicine. $3^{\text {rd }}$ ed. Baltimore (Maryland): Williams \& Wilkins, 1991:134-97.

33. Inderlied CB, Salfinger M. Antimicrobial agents and susceptjbility tests: Mycobacteria. In: Murray PR, editor. Manual of clinical microbiology. $6^{\text {th }}$ ed. Washington (District Columbia): ASM Press, 1995:1385-1404.

34. Rüsch-Gerdes S. Bestimmung der minimalen Hemmkonzentrationenvon Chemotherapeutika mit verschiedenen Methoden bei Mycobacterium avium. Pneumologie 1993;47:497-500.

35. Youmans AS, Youmans GP. The effect of "Tween 80 " in vitro on the bacteriostatic activity of twenty compounds for Mycobacterium tuberculosis. J Bacteriol 1948;56:245-52.

36. Williams DL, Waguespack C, Eisenach K, Crawford JT, Portaels F, Salfinger M, Nolan CM, Abe C, Sticht-Groh V, Gillis TP. Characterization of rifampicin resistance in pathogenic mycobacteria. Antimicrob Agents Chemother 1994;38:2380-6.

37. Canetti G, Fox W, Khomenko A, Mahler HT, Menon NK, Mitchinson DA, Rist N, Smelev NA. Advances in Techniques of testing mycobacterial drug sensitivity, and the use of sensitivity tests in tuberculosis control programmes. Bull Wld Hith Org 1969;41:21-43. 